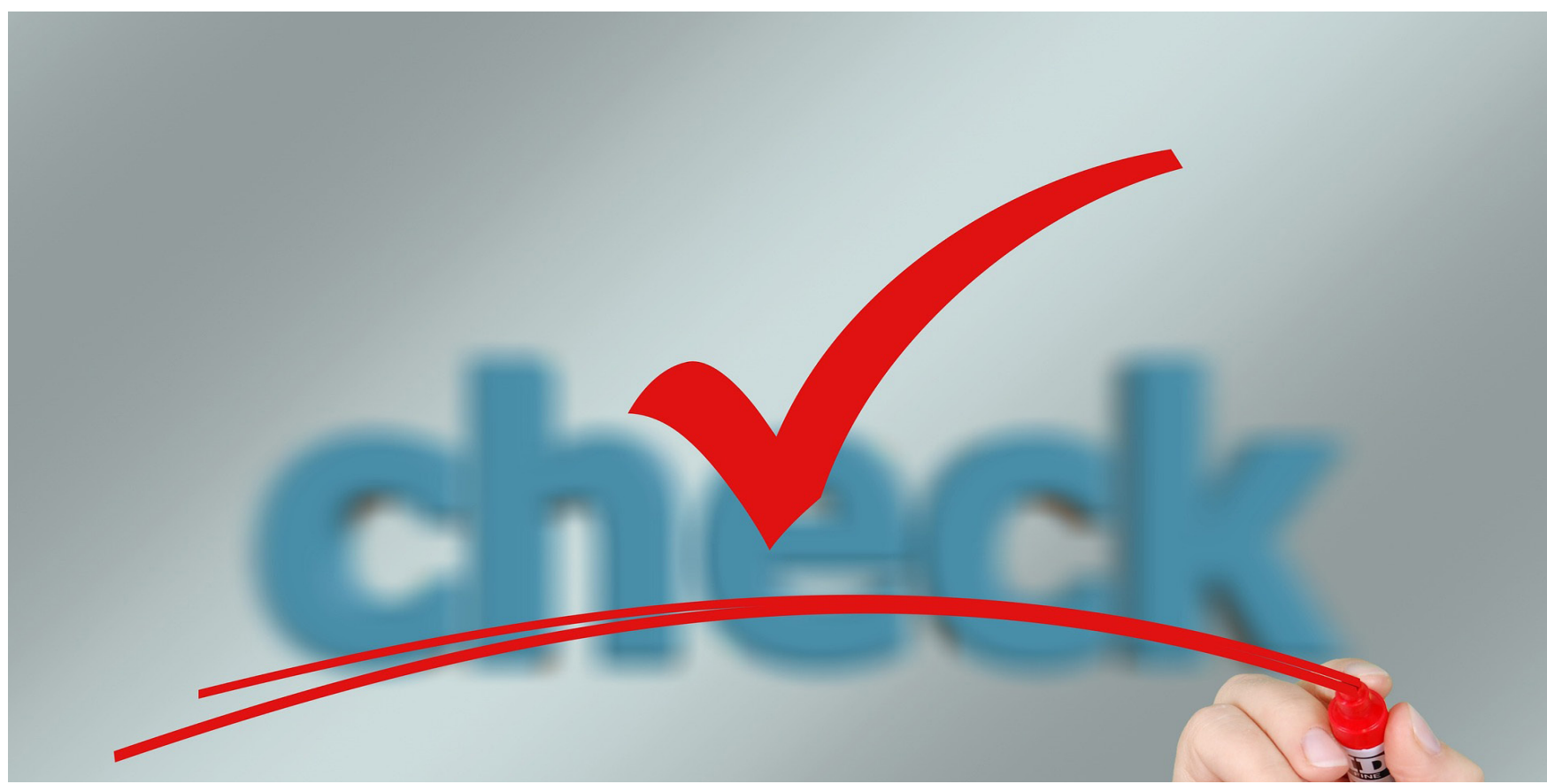

\title{
Análisis de la gestión de inventarios en la empresa ferretería la casita SAS, en Cúcuta
}

\section{Analysis of inventory management in the company ferretería La Casita SAS, In Cúcuta}

\section{Carmen Roció Cruz-Contreras}

Estudiantes Programa Contaduría Pública, carmenrociocc@ ufps.edu.co, Universidad Francisco de Paula Santander, Cúcuta, Colombia

\section{María Esmeralda Contreras-Cáceres}

Magister en Educación Investigación y Docencia Universitaria, Universidad Francisco de Paula Santander, Cúcuta, Colombia

Fabio Alonso Pérez-Berbesi

Estudiantes Programa Contaduría Pública, fabioalonsopb@ ufps.edu.co, Universidad Francisco de Paula Santander, Cúcuta, Colombia 
Cómo citar: Cruz-Contreras, C.R. , Pérez-Berbesi, F.A. \& Contreras-Cáceres, M.E. (2019). Análisis de la gestión de inventarios en la empresa ferretería la casita SAS, en Cúcuta. Reflexiones contables (Cúcuta), 2 (2), 77-86.

\section{Resumen}

Los inventarios representan para la empresa el recurso disponible más valioso por el cual la empresa debe tratar de reponer constantemente para poder satisfacer la clientela; de allí depende que llevar una gestión administrativamente confiable, dependiendo del sistema que se aplique se tendrán óptimos resultados y se reducirán los costos; dado que el caso que nos ocupa es una empresa de ferretería, tiene sus características propias en cada diversidad de productos. El trabajo que se pretende realizar, lleva por Objetivo General: Analizar la gestión de inventarios de la Ferretería La Casita SAS, en Cúcuta; Se aplicó una investigación descriptiva, documentada en revisión de trabajos de investigación que fueron previamente plasmados en una matriz constructiva para la revisión de los datos aportados en este trabajo. Se concluye que las empresas de ferretería, son muy complejos en la cantidad de productos para llevar un inventario detallado, los costos pueden variar de producto en producto, teniendo que realizar métodos de costo promedio pondera, también se aplican métodos FIFO, que es uno de los más corrientes utilizados en este tipo de inventarios.

Palabras clave: Inventarios, gestión, empresa, análisis, métodos.

\section{Abstract}

The inventories represent for the company the most valuable available resource for which the company must try to constantly replace to be able to satisfy the clientele; of there it depends that to take a management administratively reliable, depending on the system that is applied will have optimal results and will reduce the costs; since the case that occupies to us is a company of hardware store, has its own characteristics in each diversity of products. The work to be done has the following general objective: To analyze the inventory management of Ferreteria La Casita SAS, in Cúcuta. We applied a descriptive research, documented in a review of research papers that were previously reflected in a constructive matrix for the review of data provided in this work. It was concluded that hardware companies are very complex in the amount of products to keep a detailed inventory, costs may vary from product to product, having to perform weighted average cost methods, also apply FIFO methods, which is one of the most common used in this type of inventory.

Keywords: Inventories, management, company, analysis, methods. 


\section{Introducción}

El origen de los inventarios radica desde los antiguos egipcios, cuando éstos necesitaban contar el grano de las cosechas para dar abastecimiento al pueblo y se almacenaba en depósitos en tiempos de sequía. Pues no difiere mucho desde ese entonces, la utilidad que se le preste al almacenamiento de productos, para cuando exista escasez de materia prima o varíen los costos en algunos de los productos. Asimismo, la gestión de los inventarios en una empresa ferretera, tiene sus ventajas como desventajas, entre las ventajas se pueden mencionar, para poder controlarlos es necesario llevar un buen sistema, ya que el gerente al tener control pleno sobre sus entradas y salidas no tendría problema alguno en cuanto a los productos que se requieran para el stock de los mismos, su rotación, cuáles son los productos que van a salir primero. (Arciniegas \& Pantoja, 2018)

Y por otra parte, cuando el sistema no es bien manejado por la gerencia de la empresa, se presentan problemas en el almacenamiento de los mismos, Aquí se dan casos que puede presentar la empresa:

Siendo el inventario uno de los recursos más preciados de la empresa, su aprovechamiento debe ser con eficiencia, ya que el buen uso de estos reducirá en costos en la empresa; y por ende determinará el cumplimiento de la demanda de materiales y/o materia prima según sea el tipo de actividad.

Igualmente un estricto control de las entradas y salidas de inventario, permiten que no se agoten en almacén, porque la insuficiencia de productos trae retrasos en las entrega de los productos vendidos o productos terminados para la disponibilidad de las ventas; también suele suceder que los productos se vuelvan obsoletos, debido a que no tienen salida: pasaron de moda, cambio de tecnología, nunca se exhibieron para la venta, el paso del tiempo corroe los materiales, entre otros factores que afectan los materiales para la venta. (Sellán Montiel, 2019)

Pero no solamente, son materiales a disponibilidad del almacenista, hace falta la disposición del gerente de planta, quien es en línea directa el encargado de llevar este tipo de inventarios, o sea que requiere más de astucia para que el gerente cuide de los materiales dispuestos para ser consumidos, transformados, en parte o en su totalidad; aunque muchos de estos materiales nuevos que se consideren en obsolescencia o usados y que se consideren en desuso, pueden ser recuperables, porque pueden formar parte de otras piezas para ser vendidos como nuevos. (Rojas \& Zamora, 2018)

Aquí juega un papel importante el costo de la materia prima, los materiales tienden a subir o bajar de precio dependiendo de los factores que afecten el inventario; asimismo, para llevar un control también existe programas basados en la tecnología (Vélez, Pérez, Lizarazo, Alvarez, \& Ovalles, 2017), que ayudan a que el inventario sea llevado conforme a los software establecidos que brindan absoluta confiabilidad, lo cual es una solución para la gestión de negocios. (Vega, 2016); (Castro, 2018)

Por todo lo anteriormente expuesto, se formulan las siguientes interrogantes: ¿Qué ventajas tiene para la empresa Ferretería La Casita SAS, llevar un sistema de inventarios donde se apliquen los métodos adecuados para la gestión de registros del mismo?

Para dar respuesta a este interrogante, se plantea el Objetivo General: Analizar la gestión de inventarios de la Ferretería La Casita SAS, en Cúcuta; y se presentan los objetivos específicos: a) Describir los métodos aplicados para la contabilización de los inventarios; b) Examinar 


\section{0}

los costos que se asocian al inventario, que afectan los productos por diferentes factores; c) Analizar los problemas que presenta la empresa ferretera con respecto a la mala praxis de los inventarios.

Igualmente se justifica la investigación, ya que es relevante el tema de los inventarios porque se pretende analizar la gestión de los inventarios de una empresa de ferretería, la cual tiene infinidad de artículos en materias primas, en productos en proceso y en productos terminados; se pretende igualmente dar una descripción de los métodos aplicados para la contabilización de los inventarios; se examinaron los costos que se asocian al inventario y que afectan los productos por diferentes factores; como también se analizaron los problemas que presenta la empresa cuando se realiza una mala praxis de la forma como se gestionan los mismos.

Asimismo se justifica en su aspecto práctico, brindará a investigadores interesados en el tema, a través de los conocimientos, para la aplicación correcta de la contabilización y el control del sistema de inventarios en empresas del ramo ferretero; en cuanto al aspecto teórico, se justifica ya que de los trabajos analizados coadyuvarán en el desarrollo de trabajos

\section{Desarrollo}

Los inventarios, a pesar de pensar en una gran cantidad de productos almacenados, exhibidos para la venta, hay que reponerlos para que no hayan faltantes, aunque muchas veces, éstos productos sufren pérdidas, con base a la naturaleza del negocio, pero para ello es necesario aplicar métodos estándares y variables, sobre los inventarios, con la finalidad de minimizar los costos operacionales (Otiniano Collantes, 2020); y poder dar una correcta contabilización a los mismos; por tanto es necesario determinar la conceptualización de algunos términos:

\section{Inventarios:}

Con base a la funcionabilidad de los mismos, son las principales herramientas que se disponen en almacén o depósito, para ser utilizados en la medida que se vayan necesitando, ellos son materia prima, equipos, herramientas, materiales directos; (Céspedes, Paz, Jiménez, Pérez, \& Pérez, 2017); ellos contienen productos para ser transformados (productos en proceso), como también salen de allí, productos terminados con la finalidad de ser puestos a la venta; así como la trasformación de los mismos al ser manipulados. (Cardona, Orejuela, \& Rojas, 2018).

Por esta parte, es necesario en el momento del establecimiento de la empresa, el inventario es lo que marca la pauta para saber con qué activos cuenta la misma, es un factor de peso para determinar el capital que posee la empresa. (Alcedo, 2020). De allí que es necesario establecer el cumplimiento de las expectativas de la oferta y la demanda para entrar en la competencia donde opere la misma; como también aplicar la rotación de los inventarios y así obtener más flujos de efectivo para la empresa. (Suárez Gallegos \& Cárdenas, 2017).

En tal sentido es necesario que los depósitos estén suficientemente dotados de materiales para poder realizar la gestión empresarial y la comercialización de sus productos, lograr el mejor procedimiento y control de los mismos, (Ortiz-Triana, K., \& Caicedo-Rolón, 2015), dependiendo si la empresa sea grande o pequeña, el control interno será mayor o en menor escala. (Gil, 2019), (Vargas, 2018); (Bonilla, 2019). 


\section{Tipos de Inventarios}

Existen dos tipos de inventarios: inicial y final, del inventario final se resta el inventario inicial y se le suman las compras para poder conocer la existencia de mercancías; lo cual se explicará más adelante.

a) El inventario inicial son los productos que existen en la empresa, en una fecha determinada, que por lo general es un año antes del cierre del ejercicio; donde se recuentan los productos y se determina el costo de ellos.

b) Inventario final. Es el que se realiza al finalizar el ejercicio económico, luego de un año fiscal, se le suman las compras del ejercicio; se realiza una comparación con el inventario inicial para conocer el dividendo o las desventajas que se tengan en el año. (VirgUez Sierra \& Rodríguez Peña, 2019)

\section{Los inventarios se pueden subdividir en:}

a) Inventario intermitente; cuando es necesario realizar varios conteos de productos en el año, motivado a que se realizan cambios de productos de lugar; por ejemplo una nueva sucursal, en la que se requiere trasladar los productos a la nueva sede.

b) Inventario perpetuo, es el que se efectúa con base a que los materiales o la materia prima sea establecido su costo, el valor que arroja el inventario y la utilidad del mismo.

c) Inventario de materias primas; aquí se utiliza para conocer los productos primarios con que se elaborarán los nuevos productos o productos en proceso. La empresa necesita conocer con cuánto inventario de materia prima dispone para la producción.

d) Inventario de productos terminados: es necesario conocer el costo, tanto la existencia de los productos terminados y los que aún están en proceso. e) Inventario de suministros de fábrica

f) Inventario de productos en tránsito

g) Inventario de ciclo

h) Inventario de seguridad

i) Inventario de previsión de productos

j) Inventario de desacoplamiento

k) Inventario de existencias obsoletas.

\section{Métodos para la contabilización de inventarios.}

Los modelos de inventarios para la empresa son útiles porque a raíz de un diagnóstico interno, se puede verificar lo que la misma requiere, analizando cada uno de ellos, se consigue el modelo de gestión más adecuado para la misma. (Martínez Aragón, 2019)

Método ABC, es un método aplicable a los inventarios, pero que es ideal cuando la empresa no tiene ningún control, ni tiene un modelo estandarizado para realizar sus compras, lo cual se presentan problemas al momento de realizarlas porque no se sabe a ciencia cierta, qué cantidad de existencias hay del mismo material, (Betancourt, Fandiño, \& Gutiérrez, 2019),

Este método consiste en una técnica de segmentación de productos según el orden de importancia, en tres categorías, va desde la más alta "A", luego la mediana categoría "B" y por último la que menos se vende o se utiliza " $C$ ", siguiendo el principio de Pareto sus porcentajes son aplicables a los objetivos que plantea la organización de que los productos sean vendidos o utilizables todos en su mayor parte. El gerente a cuenta de esta importancia porque conoce qué productos tiene la última prioridad " $\mathrm{C}$ ". 


\section{2}

Inventario tipo multicriterio difuso, AHP que son donde se asientan tipos de inventarios que garantizan mayor efectividad para las ventas; donde es necesario seleccionar los criterios más importantes para los gerentes (incluye: subgerentes, almacenistas, jefes de compras, ventas, caja).

Pues la tradicional aplicación de los sistemas $A B C$ sólo toma en cuenta el volumen de ventas, sin la aplicación a los sujetos que están directamente relacionados con los inventarios.

En la figura siguiente se muestra la clasificación de los inventarios multicriterios, el cual está subdivido en pasos que deberán cumplirse:

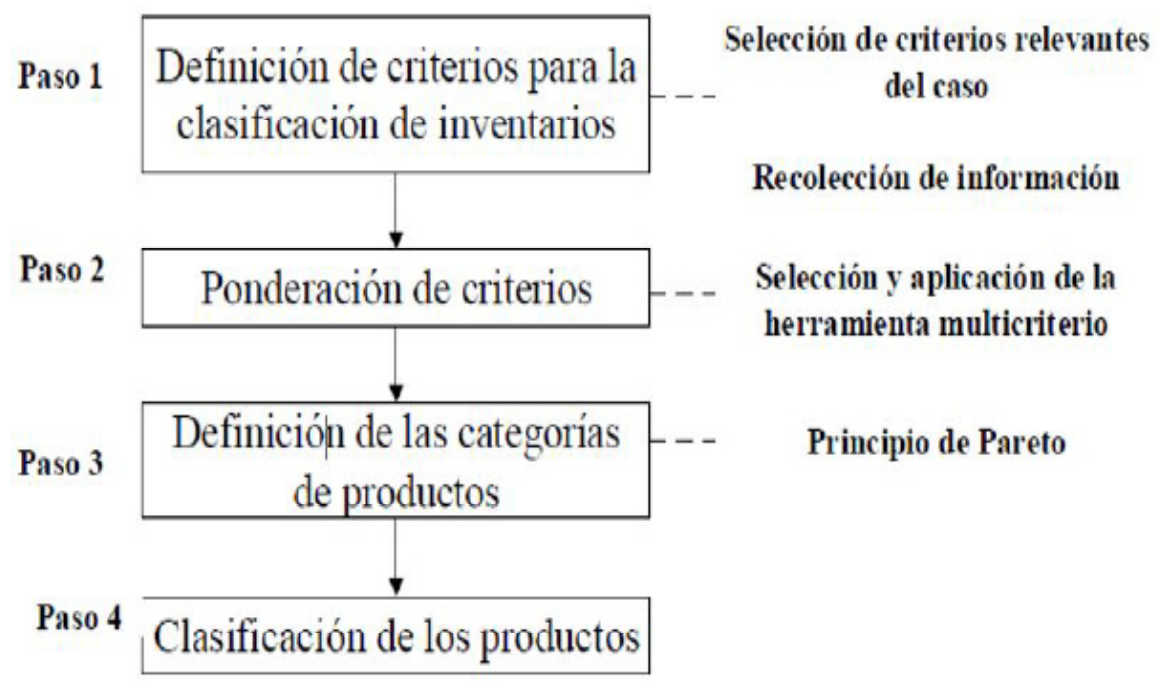

Figura 1. Método de inventarios AHP multicriterios.

Fuente: (Paredes-Rodríguez, A., Chud-pantoja, \& Osorio, 2019).

Método EOQ (Cantidad económica de pedido), el cual presenta demandas parcialmente acumulables, ejemplo típico de la cantidad de autos para la venta con cantidades suficientes para la demanda, no debería haber faltante, ni el cliente esperar a que llegue el carro del exterior o de otro Departamento; este tipo de inventario tiene éxito cuando la demanda de venta es suficiente; y para cumplir con el éxito en este tipo de inventarios, es satisfacer al cliente, mínimo costo de operación, mínima inversión en los pedidos; dado que un auto o artículo que se solicite en compra hoy, no tiene el mismo precio de mañana, los artículos tienden a subir.

Método PEPS o FIFO, primeras entradas y primeras salidas, es comúnmente utilizado por los empresarios, sobre todo en empresas que necesiten salir de los materiales que tienen más tiempo en el almacén. (Mendoza Gómez, Yescas, Aguilar Chiñas, \& Morales Pérez, 2019) Por lo general los materiales ferreteros tienen que salir lo más rápido posible, porque muchos de ellos tienen un tiempo de vencimiento; caso: las pinturas, también si son metales, pueden corroerse.

Método UEPS o LIFO, este método tiene como primera medida determinar que los últimos materiales en entrar al almacén son los primeros en salir a la producción o venta. Es un sistema que los gerentes utilizan menos, pero que no se envejecen ni pierden el valor. 
La mayoría de los materiales por este método se caducan con facilidad, porque presenta inconvenientes, al moverlos de lugar, puede descomponerse por la corrosión del tiempo, lo cual hace que se pierda el producto; también puede ser recuperable si pasa a ser parte de otro producto en vías de proceso.

Cuando el producto se descompone en el stand, supone un costo adicional, mantenerlo allí sin necesidad de ser rotado, el cual pierde su valor, su tecnología, su durabilidad si se tratara de un producto de consumo animal o humano.

Este método de incluye beneficios y desventajas, el lifo, s muy complejo, para materiales como arena, materiales de construcción, bloques para la construcción, que son homogéneos, resistentes al tiempo si están en estado de conservación, porque si la arena estuviese al sol y al gua y a ras del piso, ésta podría disminuir su cantidad; es un modelo casual en que su diseño, color, textura, calidad, no son variables en el tiempo.

Método Promedio ponderado, es uno de los métodos con que se valúa el inventario para su valoración por parte de los gerentes, muy utilizado para obtener los costos de ventas, cuando se le da continuidad a este tipo de inventarios.

Este método funciona a partir de que el comerciante genera un costo para poder adquirir el artículo, lo cual es un beneficio porque todos los artículos cuestan lo mismo, pero con respecto a la compra y el pedido que se realice, éste variará con respecto a los costos adicionales: transporte, comisiones, entre otros.

Luego lo que se puede hacer para calcularle el costo al producto es ponderar el promedio, se suman los valores del inventario para ese producto, con los inventarios de la compra nueva, luego hay que dividirlo entre el número de artículos que existen, incluidos los que existían inicialmente y los de la compra nueva. Es decir, todos los artículos.

Este método de inventarios ofrece un equilibrio preciso, porque da una visión de un promedio de costo que se pagó por la adquisición para la construcción del inventario.

A continuación un ejemplo para visualizar mejor el comportamiento del producto:

\section{Costos Asociados a los Inventarios}

Existen costos al solicitar la acumulación de materiales, ellos son relativamente altos, cuando se trata de que los productos se mantengan en un lugar específico, el depósito por ejemplo. (Torres, 2020). Algunos de estos costos son y se explicarán seguidamente:

a) Costos de pedir al proveedor

b) Costos de sostenimiento

c) Costos de agotamiento

Existen problemas cuando se disminuyen los costos de los productos; suele ser las materias primas se dañen, sean obsoletos, no se vendan debido a que están en desuso, existen el mismo productos pero con diferente tecnología; entre otros factores que aquejan al inventario. (Arango, 2011), (Nail, 2016).

\section{Resultados}

Como resultados sobre el análisis de la gestión de inventarios de la Ferretería La Casita SAS, en Cúcuta; como respuesta al primer objetivo específico sobre los métodos aplicados para la contabilización de los inventarios se tiene el método $A B C$, donde se ordenan los productos por el orden de importancia, a medida de los que más se van 


\section{4}

vendiendo primero, el multicriterio difuso, donde se da más importancia a las personas, gerentes, subgerentes; en el mismo se cumplen cuatro pasos para elaborar sus inventarios: a) se definen los criterios para su elaboración, b) se ponderan los mismos, c) se aplica una definición de categorías a los productos; y d) se clasifican los productos. (Mosquera \& Restrepo, 2016).

Igualmente se aprecia el método $\mathrm{EOQ}$, que es el que satisface la cantidad suficiente de pedidos para una demanda de artículos determinados, tal como se describió arriba una empresa de ferretería que sólo podría vender cemento, no se podría acumular porque se vencería en el tiempo.

Al examinar los costos que se asocian al inventario, se afectan los productos por diferentes factores, tales como los asociados al costo de pedir al proveedor; cuando es para el sostenimiento de los artículos, y la variabilidad de agotamiento.

Finalmente, al analizar los problemas que presenta la empresa ferretera con respecto a la mala praxis de los inventarios, éste sistema puede ser afectado en los demás departamentos: inventarios como primera medida, ventas, caja, compras; y por ende el de contabilidad, porque al estar afectado el inventario por la mala gestión y praxis, los resultados de la contabilidad y estados financieros no serían reales.

\section{Conclusiones}

La práctica común de los comerciantes es contar su inventario para poder determinar el costo de estos, en este conteo se deberá determinar cuáles están en stock, en almacén y los que estén en tránsito. Asimismo, es normal contar los productos cada vez que se requiera la realización de aplicar los estados más confiables, pueden ser, para el balance, trimestral, cada seis meses o cada año; siempre se ha de partir con un inventario inicial y uno final.

Del control estricto que se prevea llevar en la empresa, dependerá el éxito de la organización en la gestión de inventarios. Un control deficiente afectará las demás áreas de la organización, tanto la producción en lo que respecta a las ventas; ejemplo, no se podría fabricar un producto partiendo de una inexistencia, cuando realmente la hay; por lo tanto, eso redundará en costos innecesarios al momento de producir.

Una mala gestión en inventarios, cuando se considere que un producto no existe, conlleva a que el cliente pueda verse molesto y por tato no retornar al sitio de venta, por el faltante de los productos; la mala gestión radica también, en otros términos, pues los departamentos pueden verse afectados y el de contabilidad, sus estados financieros no serían reales como tal, estarían adulterados.

\section{Referencias}

Alcedo, L. (2020). La gestión de inventarios y el desempeño comercial de la empresa Líder Cloud Huánuco. Perú. Obtenido de https:// alicia.concy tec.gob.pe/vufind/Record/ UDHR_0f82c88afd688686936e622a2a2979f5

Arango, C. (2011). Creación e implementación de un programa en excel parala gestión de administración y manejo de inbentarios de repuestos en la empresa de trnasporte de mercancía TCC. Eafit, Colombia. Obtenido de https://repository.eafit.edu.co/ handle/10784/4494

Arciniegas, O., \& Pantoja, M. (s.f.). Análisis de la gestión de inventarios de las clínicas odontológicas de la ciudad de Ibarra. Holopraxis. Obtenido de https://www.revistaholopraxis. com/index.php/ojs/article/view/56/26

Betancourt, M., Fandiño, S., \& Gutiérrez, I. O. 
(2019). Diseño de un sistma de control de inventarios de productos para la empresa FerroNeumática Ltda de la ciudad de Cali. Univ. Javeriana, Colombia. Obtenido de http://vitela.javerianacali.edu.co/ handle/11522/11391

Bonilla, K. (2019). Implemntación del control interno para la gestión de inventarios de una empresa comercial. Udep, Perú. Obtenido de https://pirhua.udep.edu.pe/ handle/11042/4304?locale-attribute $=\mathrm{es}$

Cardona, J., Orejuela, J., \& Rojas, C. (2018). Gestión de inventario y almacenamiento de materias primas en el sector de alimentos concentrados. Obtenido de doi:https:// revistas.eia.edu.co/index.php/reveia/article/ view/1066

Castro, T. (2018). La importancia de la información de inventarios para la gestión financiera exitosa en la compañía "Instrumentos Médicos de Colombia SAS (IMC)". Uniagustiniana, Colombia. Obtenido de http://repositorio.uniagustiniana.edu.co/ handle/123456789/704

Céspedes, N., Paz, J., Jiménez, F. E., Pérez, L., \& Pérez, Y. (2017). La administración de los inventarios en el marco de la administración financiera a corto plazo. Redipe. Obtenido de https://revista.redipe.org/index.php/1/article/ view/272

Gil, D. (2019). El control interno y su influencia en la gestión de inventarios de las mico y pequeñas empresas comerciales del Perú, caso de la empresa Diammi GM S.A.C. de Chimbote. Uladch, Perú. Obtenido de http://repositorio.uladech.edu.pe/ handle/123456789/15679

Martínez Aragón, A. F. (2019). Fortalecimiento empresarial en el área de inventarios de la empresa Ferrelectricos Restrepo. Uiversidad
USTA, Colombia. Obtenido de https:// repository.usta.edu.co/handle/11634/21182

Mendoza Gómez, L. T., Yescas, R. V., Aguilar Chiñas, C. D., \& Morales Pérez, A. (2019). La impoortancia del control de inventario interno para el área de Boutique Deli del Hotel Marina El Cid Spa \& Beach Resort Cancún Riviera Maya. Eumed. Obtenido de https://www. eumed.net/actas/19/desarrollo-empresarial/4la-importancia-del-control-de-inventariointerno.pdf

Mosquera, D., \& Restrepo, N. (2016). Diseño de un modelo de inventario para la optimización del abastcimiento de los repuestos de la empresa mercavil, S.A. en Medellín -2015-2016. Medellín, Colombia.

Nail, A. (2016). Propuesta de mejora para la gestión de inventarios de la sociedad Repuestos España Ltda. Uach, Chile. Obtenido de http://cybertesis. uach.cl/tesis/uach/2016/bpmfcin156p/doc/ bpmfcin156p.pdf

Ortiz-Triana, K., V., \& Caicedo-Rolón, Á. J. (2015). Procedimeinto para la programación y control de la producción de una pequeña empresa. Obtenido de http://revistas.ubiobio.cl/index. php/RI/article/view/1917

Otiniano Collantes, Y. K. (2020). Diseño de un sistema de gestión de inventarios para disminuir los costos totales en la Distribuidora Yulissa Katherine. Upao, Perú. Obtenido de http:// repositorio.upao.edu.pe/handle/upaorep/5890

Paredes-Rodríguez, A., Chud-pantoja, L., \& Osorio, J. (2019). Sistema de control de inventarios multicriterio difuso para repuestos. UTP. Obtenido de https://revistas.utp.edu.co/ index.php/revistaciencia/article/view/22331

Rojas, H., \& Zamora, O. (2018). Gestión de inventarios y su efecto en la rentabilidad. Perú. 


\section{6}

Obtenido de https://repositorio.upn.edu.pe/ handle/11537/23489

Sellán Montiel, L. (2019). Plan de merjoas del control del inventariode mercaderias de la feerretería Joselón Ubicada en el Cantón Colimes. Ecuador. Obtenido de https:// repositorio.itb.edu.ec/handle/123456789/2112

Suárez Gallegos, G. G., \& Cárdenas, P. (2017). La rotación de los inventarios y su incidencia enel flujo de efectivo. Eumed. Obtenido de http://www.eumed.net/cursecon/ecolat/ec/2017/ inventarios-flujo-efectivo.html

Torres, J. (2020). Modelo de gestión para la optimización de inventarios en novocentro Madec. Ecuador. Obtenido de http://repositorio. utn.edu.ec/handle/123456789/10211

Vargas, R. (2018). El control interno y su influencia en la gestión de inventarios delas empresas del sector comercio del Perú. Caso empresas L\&S. Uladch, Perú. Obtenido de http://repositorio. uladech.edu.pe/handle/123456789/15616

Vega, E. (2016). Propuesta de mejoramiento para la gestión de bodega de materiales e insumos para impresoras de la empresa Coplan. Chile. Obtenido de http://repositorio.unab.cl/xmlui/ handle/ria/2755

Vélez, S., Pérez, J., Lizarazo, K., Alvarez, L., \& Ovalles, C. (2017). Inventario de software implementado en hoteles del área metropolitana de San José de Cúcuta. Obtenido de https:// www.fesc.edu.co/Revistas/OJS/index.php/ convicciones/article/view/234Obtenido de https://books.google.co.ve/s?id=657QDwAAQ BAJ\&pg $=$ PT132\&lpg $=$ PT132\&dq $=$ direcci $\%$ C3 $\% \mathrm{~B} 3 \mathrm{n}+$ estrat $\% \mathrm{C} 3 \%$ A9gica + de + la + investigaci $\% \mathrm{C} 3 \% \mathrm{~B} 3 \mathrm{n}+$ scielo + redalyc \& source $=$ bl\&ots $=\mathrm{O}$ dta9v81Zy

VirgUez Sierra, M. Y., \& Rodríguez Peña, B. A.
(2019). Evaluación del cumlimiento delárea de planeación y pronóstico de Disan Colombia S.A., 2017-2018. Uniminuto, Colombia. Obtenido de https://repository.uniminuto.edu/ handle/10656/7735 